\title{
ACTION DU CARBONATE DE CALCIUM SUR LA VASE D'UN ÉTANG \\ I - ESSAIS COMPARATIFS DANS L'ÉTANG ET AU LABORATOIRE
}

\author{
CI. MARTY* et J. SECHET **
}

\section{RÉSUMÉ}

Ce travail a pour but de mener in situ et au laboratoire des expériences à caractère quantitatif sur l'action du carbonate de calcium en apport au niveau de l'interface vaseeau de l'étang de Léon.

II permet d'observer dans les sédiments l'élévation du $\mathrm{pH}$ et des populations microbiennes aérobies en relation avec une sensible diminution des composés organiques.

Dans la phase liquide, la mise en évidence d'une meilleure oxygénation du milieu se trouve liée à une implantation d'algues benthiques dans les vases traitées.

Enfin, des analyses menées en fin d'expérience permettent d'évaluer la zone de pénétration du carbonate de calcium dans le sédiment et appellent une étude complémentaire permettant d'apprécier l'action de la craie lorsqu'elle est incorporée, à l'état divisé. dans la vase.

Mots Clés : Étang - Carbonate de calcium - Microbiologie - Oxygénation de l'eau Composés organiques.

\section{SUMMARY}

The purpose of this work was to make a quantitative study in situ and in the laboratory of the effect on the sediments of chalk spread in an experimental area of a large shallow lake, Léon pond in South West France.

A rise in $\mathrm{pH}$ and aerobic microbial populations was observed in sediments in relation to a significant decrease of organic compounds.

A better oxygenation of the liquid phase is linked with appearance of benthic algae in the treated sediments.

Finally, depth of penetration of calcium carbonate into the sediment was weak. Further study is required in order to appreciate chalk action when incorporated into the mud in particulate form.

\section{PLAN}

\section{I - INTRODUCTION}

\section{॥ - MATÉRIEL ET MÉthOdES}

2.1. Présentation et caractéristiques de l'étang

2.2. Protocole expérimental

\section{III - RÉSULTATS}

3.1. Modifications physico-chimiques
a) dans l'eau
b) dans la vase

* Laboratoire d'Écologie des Poissons et d'Aménagement des Pêches Centre de Recherches Hydrobiologiques I.N.R.A. BP $n^{\circ} 3$ SAINT PEE SUR NIVELLE 64310 ASCAIN (FRANCE)

* Laboratoire de Botanique Appliquée et de Microbiologie Université de Bordeaux I Avenue des Facultés 33405 TALENCE CÉDEX (FRANCE) 
3.2. Incidences microbiologiques

a) dans l'eau

b) dans la vase

3.3. Observations de la flore algale

IV - DISCUSSION ET CONCLUSION

\section{BIBLIOGRAPHIE}

\section{I - INTRODUCTION}

Parmi toutes les richesses mises à notre disposition, l'eau est l'une des plus importantes. Afin de sauvegarder ce patrimoine naturel, plusieurs actions sont entreprises pour la conservation ou le maintien de la qualité de cet élément. Les réserves d'eau sont encore assez nombreuses et les grands lacs landais de la région du Sud-Ouest en sont un bon exemple. Cependant, comme toute pièce d'eau, ils ont tendance à se combler et l'on dispose de techniques bien connues pour contrecarrer cette évolution.

Un nouveau mode d'intervention, par épandage de craie, est actuellement appliqué dans certaines régions, à la suite de constatations effectuées au départ en Angleterre (SAWYER, 1964, 1965, 1967 a, 1967 b, 1967 c, 1967 d)

En France, les premiers essais furent pratiqués sur les cours d'eau normands (SALES, 1970 a, 1970 b; GAGNIARD, 1965, 1966, 1968). D'autres utilisateurs ont expérimenté ce procédé dans les eaux continentales (CAVELIER, 1978; CAVELIERJACOUEMAIN, 1977 a, 1977 b; LAURENT, SECHET, 1973; LEYNAUD et al., 1969; MARTY, 1972; ROFES, 1971, 1975) ou en milieu marin (FEUILLET, GOULEAU, 1977. 1979).

Plusieurs hypothèses concernant l'action du carbonate de calcium sur la diminution de la couche de vase lors de ces épandages sont avancées. Nous citerons principalement pour les traitements en rivière LEYNAUD et al., (1969) pour qui le résultat serait dû à des processus strictement physiques (modifications de la viscosité et de l'adhérence de la vase). VAN BENEDEN et VAN BENEDEN (1966) voient une action chimique de la craie sur les bicarbonates calciques déterminant, entre autres, une transformation des substances humiques qui affaiblirait le pouvoir d'adhérence au substrat. ROFES $(1971,1973)$ impute aux ions $\mathrm{Ca}^{+}+$après leur passage en solution, la floculation des colloïdes et une diminution de la cohésion des vases.

Pour les épandages effectués en étang, les facteurs hydrodynamiques, tels que le courant, ne peuvent jouer un rôle principal. Sans exclure l'éventualité d'une action physico-chimique, MARTY (1972), LAURENT et SECHET (1973), pensent que l'activité microbienne constitue le phénomène majeur entrainant la minéralisation bénéfique de la matière organique constatée dans la vase.

Après une première étude de type qualitatif (MARTY, 1972), il était intéressant de compléter ces résultats par des essais comparatifs à caractère quantitatif sur la vase d'un étang, en essayant :

- de déterminer le traitement le plus approprié en fonction de la dose de carbonate de calcium épandue :

- de mieux préciser les phénomènes observés.

\section{II - MATÉRIEL et MÉTHOdes}

\subsection{Présentation et caractéristiques de l'étang}

L'étang de Léon, choisi pour cette étude, est l'un des plus petits de la bordure océanique de l'Aquitaine. De forme quasi circulaire, sa superficie actuelle est d'environ 230 hectares. Sa plus grande dimension atteint presque $2 \mathrm{~km}$ et sa profondeur est relativement faible $(1,50 \mathrm{~m}$ maximum pour 80 p. 100 des fonds) (fig. 1).

Grâce au rapport publié par le C.T.G.R.E.F. (1974), on peut mieux évaluer les apports et les sorties en matières solides et minérales, nitrates et phosphates de cet étang qui reçoit annuellement $160 \times 10^{6} \mathrm{~m}^{3} \mathrm{~d}$ 'eau (soit 35 fois son volume) et dont le débit de sortie est de $150 \times 10^{6} \mathrm{~m}^{3}$. Le tableau 1 regroupe les différentes valeurs indiquées en tonne/an.

Bien qu'approximatif, ce bilan permet d'apprécier la vitesse assez grande de 
comblement par matière solide et de constater qu'une grande partie des nutriments est utilisée par assimilation au niveau des végétaux, ou absorption par les sédiments.

\section{Figure 1: Etang de Léon.}

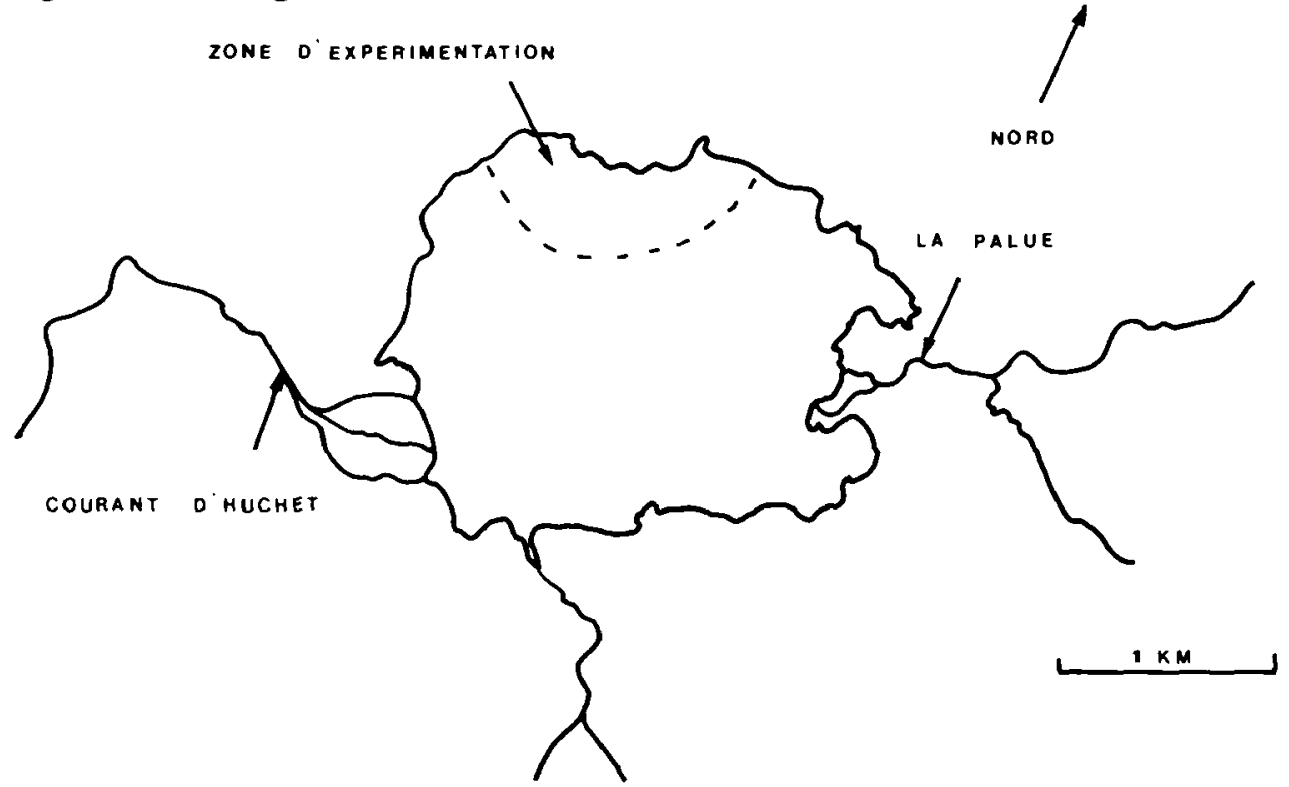

Tableau 1 : Bilan d'entrée et de sortie dans l'étang de Léon, en tonnes/an. (C.T.G.R.E.F., 1974).

\begin{tabular}{|l|c|c|c|c|c|}
\hline & $\begin{array}{l}\text { Matière en } \\
\text { suspension }\end{array}$ & $\begin{array}{c}\text { Matière en } \\
\text { charriage }\end{array}$ & $\begin{array}{c}\text { Matière } \\
\text { totale }\end{array}$ & $\begin{array}{l}\text { Quantité } \\
\text { d'azote } \\
\text { (nitrate) }\end{array}$ & $\begin{array}{l}\text { Quantité de } \\
\text { phosphore } \\
\text { (phosphate) }\end{array}$ \\
\hline Entrée & 1520 & 2490 & 4010 & 233 & 0,99 \\
Sortie & 1275 & 1690 & 2955 & 68 & 0,47 \\
\hline
\end{tabular}

\subsection{Protocole expérimental}

La craie employée dans cette expérimentation provient dugisement d'Omey, en Champagne. Calcaire naturel, d'origine planctonique, son taux de carbonate de calcium est très important (98,7 p. 100). Il est constitué de nombreux phytoflagellés (4 a $5 \mu$ ) dont les coques calcaires (coccosphères) sont composées de plaquettes particulièrement fines (ANONYME, 1965).

Dans cette série expérimentale dont le caractère commun est l'adjonction sur l'interface vase-eau de quantités différentes de carbonate de calcium, deux groupes d'expériences ont été mis en place: l'un in situ (étang de Léon), l'autre effectué au laboratoire.

Nous avons testé à côté d'un lot témoin, différents apports correspondant à 0,5, 1 , 2 et 4 fois la dose de référence, cette dernière représentant l'épandage de 5 tonnes par hectare.

Dans l'expérience ayant lieu sur l'étang de Léon, il a été délimité près de la rive Nord-Ouest 4 carrés de $25 \mathrm{~m}$ de côté, séparés entre eux par une cinquantaine de mètres. L'apport de craie fut distribué aussi uniformément que possible à partir d'une barque.

Au laboratoire, pour se rapprocher au maximum des conditions rencontrées en milieu naturel, nous avons utilisé des cylindres en plexiglass de $20 \mathrm{~cm}$ de diamètre et de $1 \mathrm{~m}$ de hauteur. Ils sont remplis sur cette hauteur $(1 \mathrm{~m})$ par moitié de vase et d'eau provenant de l'étang. Un cache noir a été positionné sur toute la hauteur de la vase, afin 
de soustraire celle-ci aux radiations lumineuses latérales. Après chargement de la phase liquide et solide, le carbonate de calcium déposé à la surface de l'eau a recouvert l'interface vase-eau.

Six expériences ont été suivies dans cette série. Elles nous ont permis d'effectuer des répétitions, d'intervenir à des fréquences différentes de prélèvement, ou de tester l'effet d'un facteur particulier: adjonction d'un éclairage supplémentaire pouvant accélérer certains phénomènes (MARTY, 1981).

Les paramètres physico-chimiques suivants : température, $\mathrm{pH}$. oxygène dissous, azote total. carbone organique et matière organique, ont été suivis tout au long de l'expérience par des mesures classiques (MARTY, 1981). Parmi celles-ci, nous signalerons pour le dosage de l'azote total, du carbone organique et de la matière organique, la mise en cuvre respectivement des méthodes de KJELDAHL (DUCHAUFOUR, 1965), d'ANNE modifiée, et de perte au feu.

Quant aux analyses microbiologiques qui ont permis de quantifier les populations bactériennes dans l'eau et la vase, on procède par la méthode classique des suspensions-dilutions, et l'ensemencement d'une série de tubes contenant un milieu liquide approprié aux réactions spécifiques du groupe de microorganismes dont on veut déterminer la population (POCHON et TARDIEUX, 1962). Les deux groupes physiologiques suivis lors de ces ensemencements sont

- les ammonifiants dans l'eau et la vase, dont l'importance peut être considérée comme révélatrice de la "microflure totale".

- les minéralisateurs du soufre organique dans la vase.

Pour pouvoir comparer les différentes moyennes arithmétiques, nous avons utilisé la méthode statistique, appliquée au cas des petits nombres d'échantillons, du test de $t$ de STUDENT.

Lorsque les résultats comparés par cette méthode seront significativement différents, ils seront accompagnés des signes + et ++ correspondant à des coefficients de sécurité égaux à 95 et 99 pour cent.

\section{III - RÉsultats}

\subsection{Modifications physico-chimiques}

a) dans l'eau

En étang, les emplacements traités étant relativement proches les uns des autres et le volume d'eau important, peu de variations sont observées en fonction des points de prélèvement. C'est ansi que la dureté totale et le $\mathrm{pH}$ restent quasiment stables autour des valeurs égales à $28 \mathrm{mg} / \mathrm{l}$ et 6,9 unité $\mathrm{pH}$.

De même pour les valeurs de l'oxygène dissous, les moyennes arithmétiques de ce facteur sont proches les unes des autres, et les résultats statistiques correspondants $n$ 'indiquent pas de différences significatives. Cependant, la comparaison des courbes de ce paramètre avec celles du relevé des températures de l'eau met en évidence une similitude de tracé dont les minima et maxima sont décalés de 1 ou 2 mois (MARTY. 1981). En effet, l'élévation de la température précède la pousse algale, principale responsable de la photosynthèse en étang, donc de la production d'oxygène.

Par contre, au laboratoire, l'apport de carbonate de calcium a déterminé dans les tubes traités par rapport aux témoins une nette élévation de la dureté totale et du pH, plus sensible en été pour ce dernier paramètre (tabl. 2).

Tableau 2: Moyennes du pH et de la dureté totale de l'eau dans les bacs du laboratoire au cours des $\mathbf{3 6}$ mois d'expérimentation.

\begin{tabular}{|l|r|r|r|r|r|}
\hline Tubes & Témoin & $1 / 2$ Dose & 1 Dose & 2 Doses & 4 Doses \\
\hline $\begin{array}{c}\text { pH, en unitè } \\
\text { pH }\end{array}$ & 6,33 & $7,76^{++}$ & $8,0^{++}$ & $8,0^{++}$ & $8,05^{++}$ \\
\hline $\begin{array}{l}\text { Dureté totale } \\
\text { en mg/ }\end{array}$ & 27,8 & $79,9^{++}$ & $119,0^{++}$ & $93,4^{++}$ & $113,9^{++}$ \\
\hline
\end{tabular}


L'oxygène dissous dans l'eau des tubes traités a lui aussi subi de fortes augmentations par rapport au témoin, avec un maximum enregistré dans le tube 2 doses (tabl. 3). II s'est en effet développé tout au long de l'expérience dans les tubes traités et plus particulièrement dans le tube 2 doses une importante flore qui a permis au phénomène de photosynthèse de se manifester.

Tableau 3 : Moyennes du taux d'oxygène dissous dans l'eau des bacs du laboratoire au cours des $\mathbf{3 6}$ mois d'expérimentation.

\begin{tabular}{|c|c|c|c|c|c|}
\hline Tubes & Témoin & $1 / 2$ Dose & 1 Dose & 2 Doses & 4 Doses \\
\hline $\begin{array}{l}\text { Teneur en } \\
\text { oxygène } \\
\text { exprimée en } \\
\text { \% de la } \\
\text { saturation }\end{array}$ & 90,6 & 104,7 & 108,4 & $123,4^{+}$ & 105,4 \\
\hline
\end{tabular}

b) dans la vase

Dans les vases, le $\mathrm{pH}$ accuse une augmentation constante due à l'adjonction du carbonate de calcium (fig. 2).

Figure 2: Evolution du pH des vases dans les cylindres expérimentaux du laboratoire.

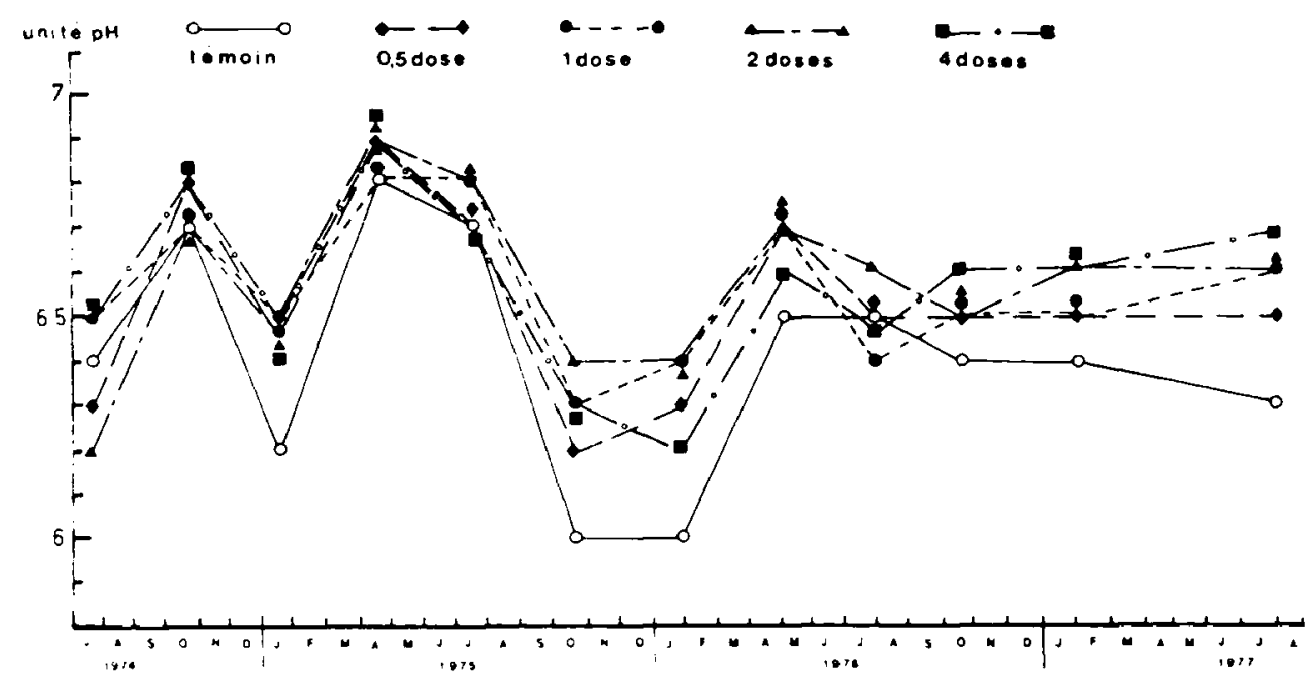

Plusieurs facteurs ont été suivis: azote total, carbone organique, matières organiques totales.

Dans l'ensemble, aussi bien in situ qu'au laboratoire, on enregistre pour les composés organiques des vases traitées, malgré quelques légères fluctuations positives.

Tableau 4 : Variations en pourcentage des composés organiques dans la vase par rapport au témoin (laboratoire).

\begin{tabular}{|l|c|c|c|c|}
\hline & $1 / 2$ Dose & 1 Dose & 2 Doses & 4 Doses \\
\hline Azote total & $-2,99$ & $-2,95$ & $+1,56$ & $-3,39$ \\
\hline $\begin{array}{l}\text { Carbone } \\
\text { organique }\end{array}$ & $-2,41$ & $-5,51$ & $-2,12$ & $-11,86$ \\
\hline $\begin{array}{l}\text { Matière } \\
\text { organique }\end{array}$ & $-2,17$ & $-7,2$ & $-2,87$ & $-5,62$ \\
\hline
\end{tabular}


une baisse des teneurs par rapport à celles enregistrées dans les vases témoins (MARTY, 1981). Si l'ampleur de ces diminutions est faible, ces dernières peuvent cependant atteindre 10 a 12 p. 100 dans les vases traitées par quatre fois la dose de référence (en laboratoire) (tabl. 4).

\subsection{Incidences microbiologiques}

a) dans l'eau

Nous retrouvons en étang pour la microflore totale la même absence de différenciation, entre les 4 stations, que celle constatée lors de l'analyse de la dureté totale et du pH de l'eau due à la fois au volume d'eau et à ses mouvements. Par contre, il est intéressant de signaler (fig. 3) une assez grande activité microbienne en hiver et en début d'automne par opposition aux populations de microorganismes dénombrées en période printanière qui se trouvent à leur niveau le plus bas.

Figure 3: Evolution du nombre de bactéries/ml de la microflore totale de

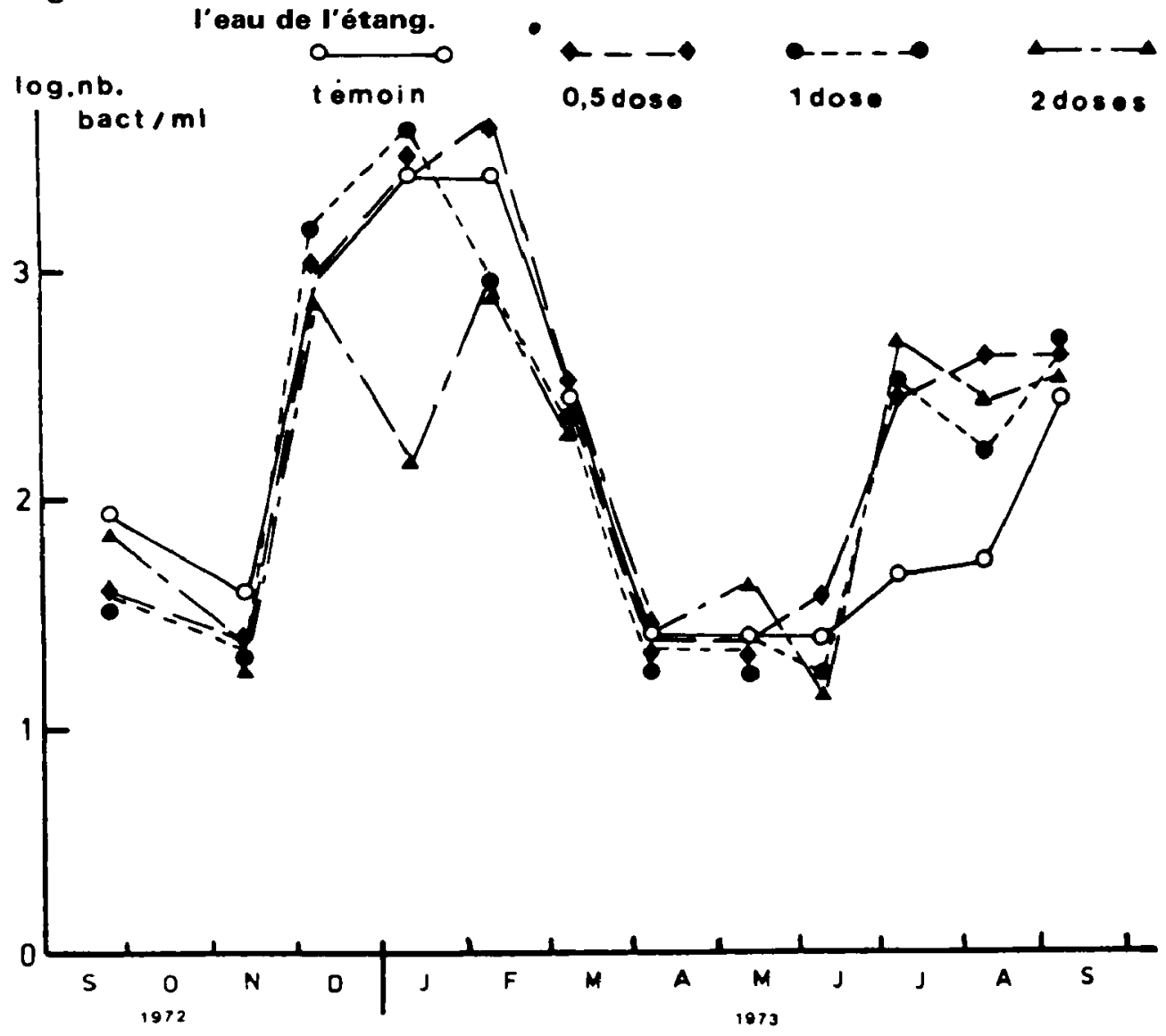

Au laboratoire, où le système expérimental est par définition isolé du milieu naturel, on observe moins nettement ce phénomène. On note par contre une sensible augmentation du nombre moyen de bactéries par gramme. La comparaison des populations existantes dans le tube 2 doses se révèle significativement différente de celle du témoin (tabl. 5) (fig. 4).

Tableau 5 : Microflore totale de l'eau (laboratoire).

Moyennes des logarithmes du nombre de germes $/ \mathrm{ml}$.

\begin{tabular}{|c|c|c|c|c|}
\hline Témoin & $1 / 2$ Dose & 1 Dose & 2 Doses & 4 Doses \\
\hline 4,30 & 4,66 & 4,41 & $4,80^{+}$ & 4,61 \\
\hline
\end{tabular}


Figure 4: Evolution du nombre de bactéries $/ \mathrm{ml}$ de la microflore totale de I'eau (laboratoire).
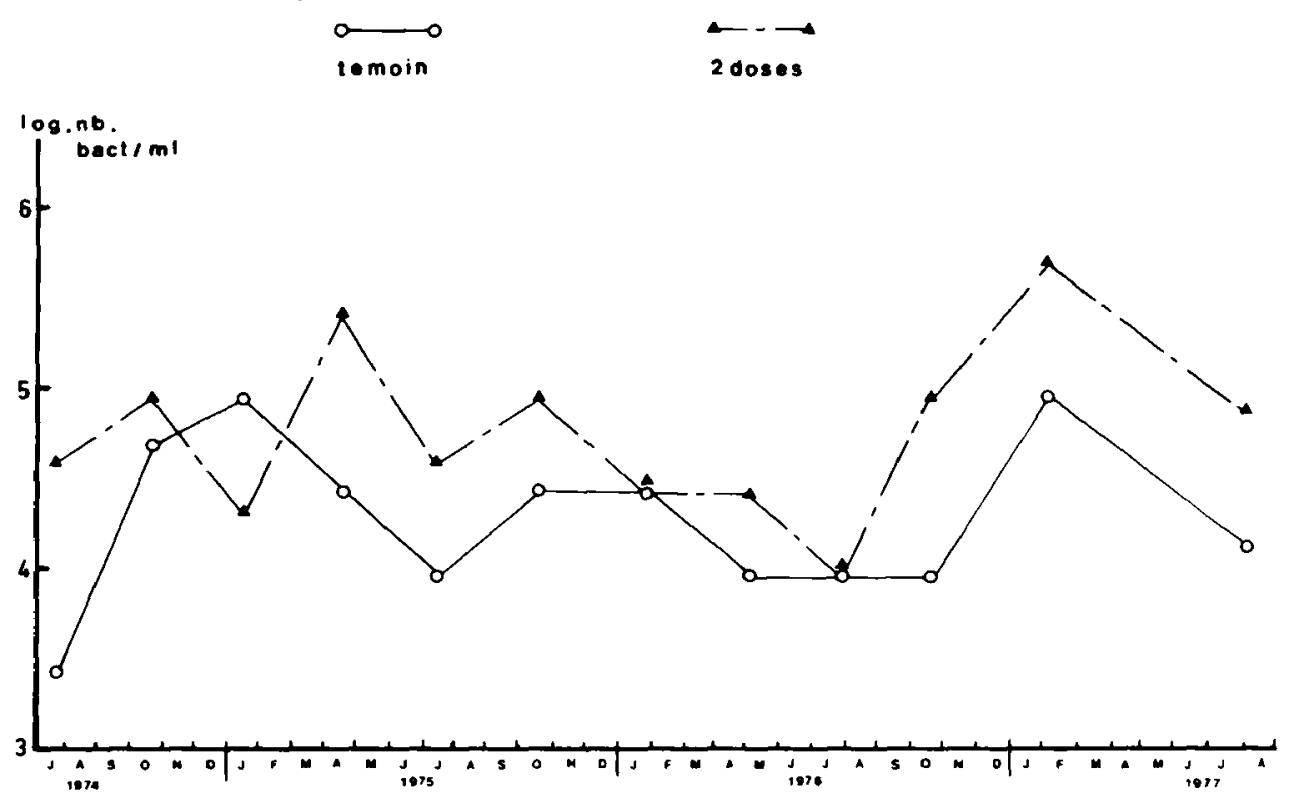

b) dans la vase

Pour les populations bactériennes représentatives de la microflore totale, qu'elles appartiennent aux vases traitées en étang ou en laboratoire, nous constatons par rapport aux vases témoins plusieurs résultats similaires:

Tableau 6 : Microflore totale des vases.

Moyennes des logarithmes du nombre de germes/g.

\begin{tabular}{|l|c|c|c|c|c|}
\hline & Témoin & $1 / 2$ Dose & 1 Dose & 2 Doses & 4 Doses \\
\hline Etang & 4,23 & 4,16 & 4,54 & 4,54 & - \\
\hline Laboratoire & 4,78 & 4,84 & 5,21 & $5,20^{+}$ & 5,12 \\
\hline
\end{tabular}

augmentation générale des valeurs moyennes du nombre de germes/g, puisque nous passons en étang de $20.10^{3}$ à $35.10^{3}$ germes $/ \mathrm{g}$ et de $60.10^{3}$ à $170.10^{3}$ au laboratoire (tabl. 6):

- très nette élévation de ces populations durant la période s'étendant de mars-avril à septembre-octobre et diminution importante de ces mêmes populations de décembre à février.

Quant aux populations anaérobies mises en évidence par l'étude des minéralisateurs du soufre organique. l'on constate en étang un faible accroissement du nombre de germes/g, d'ailleurs non significatif, dans les vases traitées par rapport au lot témoin. Pour les résultats obtenus au laboratoire concernant ce paramètre, aucune différence quantitative ne peut être mise en évidence; seules les variations saisonnières déjà observées lors des dénombrements de la microflore totale de la vase se reproduisent ici.

Après avoir suivi l'évolution des divers paramètres physico-chimiques et biologiques dans le temps, on a procédé, pour les expériences se déroulant au laboratoire, à un bilan complet d'analyse de l'eau, de la vase de surface (5 premiers centimètres) et de la vase de fond en fin d'expérimentation. Cette différenciation a pour but de tester plus particulièrement la zone d'action du carbonate de calcium. 
L'on retrouve lors des mesures pour l'ensemble des paramètres les principales observations précédemment décrites. Cependant, la comparaison des résultats obtenus suivant le niveau de prélèvement darıs la vase amène plusieurs remarques.

- des modifications sensibles sont enregistrées, d'une part entre vases trattées et vases témoins, et d'autre part entre prélèvement de surface et prélèvement de fond:

- l'élévation du $\mathrm{pH}$ de la vase et l'augmentation des populations microbiennes aérobies sont aussi marquées que la diminution des composés organiques est nette (tabl. 7).

Tableau 7 : Bilan de fin d'expérimentation (laboratoire).

\begin{tabular}{|c|c|c|c|c|c|c|}
\hline Tubes & \multicolumn{2}{|c|}{$\begin{array}{l}\text { Azote total dans } \\
\text { la vase }(g / \mathrm{kg})\end{array}$} & \multicolumn{2}{|c|}{$\begin{array}{l}\text { Carbone organique } \\
\text { dans la vase (") }\end{array}$} & \multicolumn{2}{|c|}{$\begin{array}{l}\text { Matière organique } \\
\text { dans la vase }(\because)\end{array}$} \\
\hline Prélèvement de & Surface & Fand & Surface & Fand & Surface & Fond \\
\hline Témo in & 18,42 & 22,85 & 20,94 & 19.58 & 37,60 & 40,59 \\
\hline $1 / 2$ Dose & 17,31 & 21,90 & 18,70 & 20.07 & 35,83 & 43,69 \\
\hline 1 Dose & 19,04 & 21,09 & 19,59 & 22,59 & 35,00 & 40,15 \\
\hline 2 Doses & 17,67 & 21,64 & 18,09 & 22,21 & 32,86 & 40,57 \\
\hline 4 Doses & 16,36 & 22,06 & 18,94 & 23,71 & 33,10 & 40,16 \\
\hline Tubes & $\begin{array}{l}\mathrm{pH} \text { dan } \\
\text { unite }\end{array}$ & vase & $\begin{array}{l}\text { Microfic } \\
\text { dans la }\end{array}$ & $\begin{array}{l}\text { otale } \\
+\end{array}$ & $\begin{array}{l}\text { Minéralis } \\
\text { soufre or } \\
\text { dans la }\end{array}$ & $\begin{array}{l}n \text { du } \\
\text { que } \\
+\end{array}$ \\
\hline Prèlèvement de & Surface & Fond & Surface & Fond & Surface & Fond \\
\hline Témo in & 6,5 & 6,3 & 4,39 & 3,65 & 3,06 & 3,39 \\
\hline $1 / 2$ Dose & 6,6 & 6,4 & 4.65 & 3,39 & 3,39 & 3,39 \\
\hline 1 Dose & 6,7 & 6,4 & 4,65 & 3,06 & 3,39 & 2,87 \\
\hline 2 Doses & 6,8 & 6,5 & 4,87 & 3,65 & 3,39 & 3,65 \\
\hline 4 Doses & 6,8 & 6,4 & 5,39 & 3,87 & 3,65 & 3,87 \\
\hline
\end{tabular}

+ logarithmes du nombre de germes/g.

Figure 5: Evolution du pH, de la microflore et de la matière organique lors des bilans de fin d'expérimentation au laboratoire.

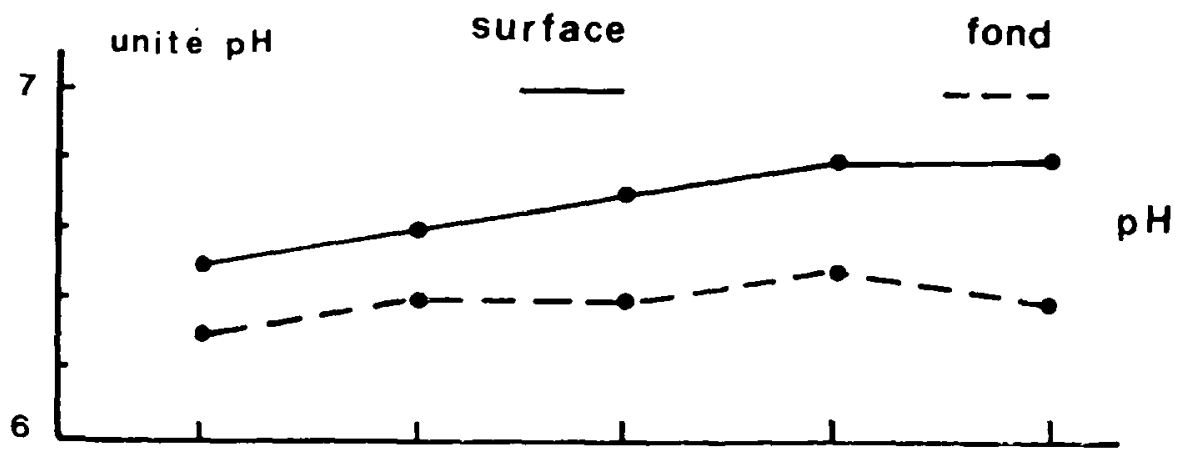


En reportant sur la figure 5 les trois graphiques représentant les valeurs obtenues lors du bilan de fin d'expérimentation pour le pH, les populations aérobies et les teneurs en matière organique, on illustre bien les principaux phénomènes observés en fonction des doses et du niveau de prélèvement.
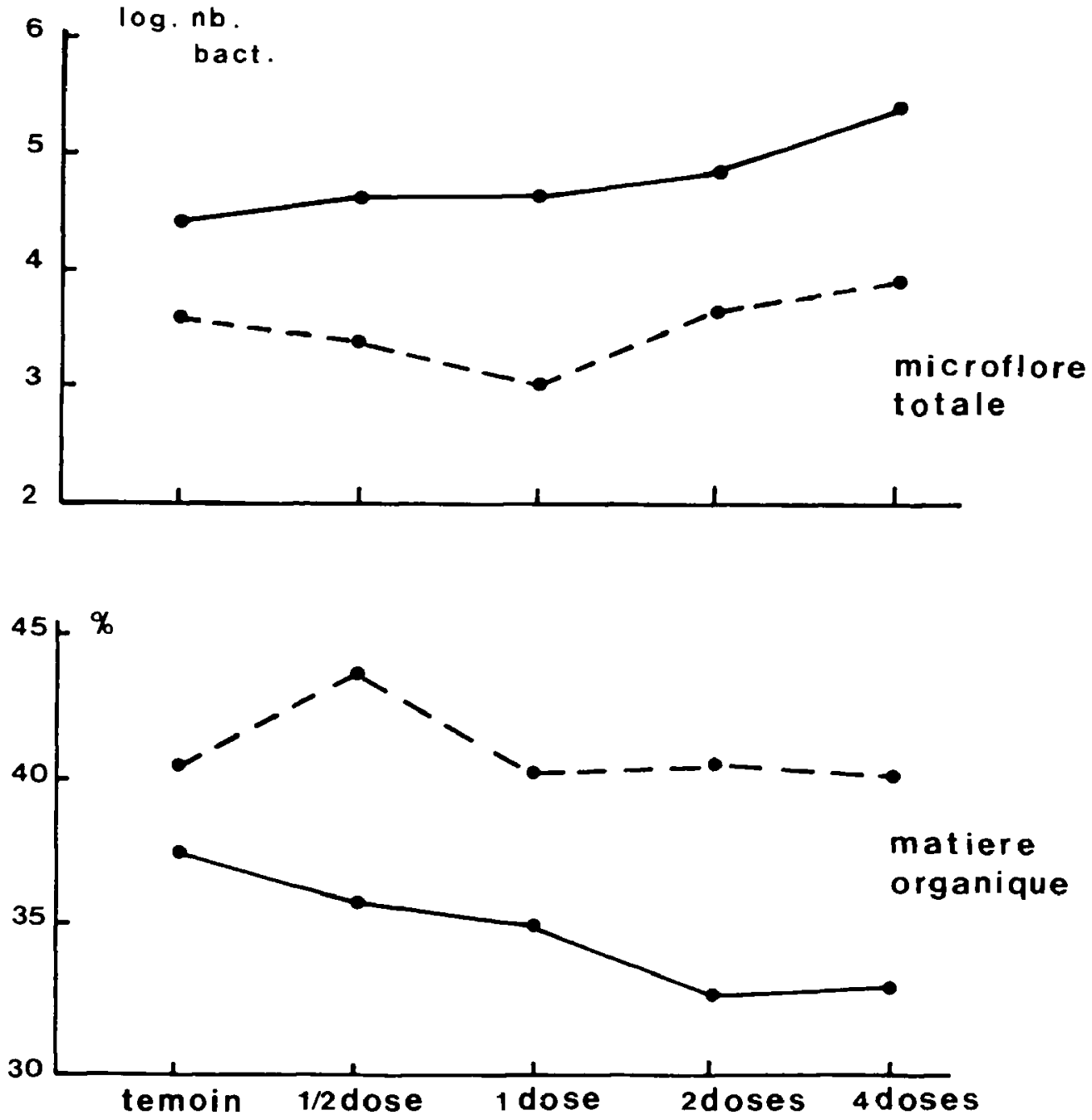

\subsection{Observation de la flore algale}

Après un délai de quelques mois, et tout au long de l'expérience, nous avons noté l'apparition puis le développement continu d'une flore algale dans l'ensemble des tubes. un maximum de densité étant enregistré dans ceux qui ont reçu une ou deux doses de carbonate de calcium, alors que peu de végétation s'est développée dans les tubes témoins.

Il est à signaler d'autre part que dans les tubes traités, l'on a observé une plus grande transparence de l'eau, ainsi qu'une fluidité plus importante des premiers centimètres de vase.

\section{IV - DISCUSSION ET CONCLUSION}

Nous venons de voir que l'adjonction de carbonate de calcium sous forme de craie pulvérulente à différentes doses dans un étang, in situ ou en milieu clos, au laboratoire, modifie sensiblement nombre de paramètres physico-chimiques ou microbiologiques. Plusieurs résultats n'appellent pas de réflexion particulière. Par contre, l'évolution de certains paramètres mérite une analyse plus précise. 
C'est le cas de l'augmentation de la valeur du $\mathrm{pH}$ de l'eau constatée durant les mois chauds au laboratoire. Ce phénomène saisonnier déjà signalé par STUDER (1974) dans les étangs de la Brenne peut être en relation avec l'activité photosynthétique, laquelle réduit le taux de $\mathrm{CO}_{2}$ et corrélativement la concentration en $\mathrm{H}^{+}$(NISBET et VERNEAUX, 1970; HUSSENOT, 1972; BERTRU, 1975; GRYGIEREK et WASILEWSKA, 1979).

De même, selon les données classiques, on aurait pu s'attendre lors du dénombrement de la microflore totale de l'eau de l'étang à une élévation des populations de microorganismes au printemps et en automne, ce qui est presque le cas de l'étang de Cazaux (CHARTRAIN-DEMARQUEZ, 1977 ; DEMARQUEZ et SECHET, 1977); or, cette augmentation in situ a bien lieu fin d'été - début automne, mais elle est plus importante en hiver.

Trois interprétations peuvent être envisagées

- La population microbienne en été est anormalement basse et aurait dû se trouver au-dessus du niveau atteint en hiver. L'étang ceinturé par la forêt et la pousse algale étant à son maximum, il peut y avoir inhibition de la microflore due à des substances toxiques, tels certains exsudats racinaires ou produits provenant de la dégradation biologique de résidus végétaux. Ce sont des phénomènes jusqu'à présent $\mathrm{mal}$ connus, mais fréquemment signalés, notamment en forêt.

- L'augmentation de la population algale, à son maximum en été, joue un rôle de piège à nutriments, privant ainsi les populations de microorganismes des éléments nécessaires à leur croissance

- La microflore totale de l'eau dans l'étang atteint son maximum naturellement en fin d'été - début d'automne et en hiver. Dans ce cas, ces variations sont liées comme certains auteurs le signalent, soit à des variations saisonnières, soit à des facteurs autres que la température; dans les sols, parmi ces facteurs déterminants pour l'évolution des populations microbiennes, une forte humidité, un important apport de matière organique, etc... peuvent être envisagés. RAKNHO (1963) et ASKEL (1965), étudiant des sols où le taux d'humidité est élevé, notent une augmentation de la population des microorganismes en hiver.

De ces trois hypothèses, celle où la flore algale peut jouer le rôle de piège à nutriment pourrait être retenue comme valable pour expliquer la baisse, constatée en été, des populations de la microflore dans l'eau. En effet, dans les expériences de laboratoire où l'on ne peut retenir l'action inhibitrice d'exsudats racinaires provenant de la forêt, ou l'apport important de matières organiques, nous retrouvons partiellement ce phénomène.

II est particulièrement sensible dans le tube 2 doses (fig. 4) où le taux d'oxygène dissous dans l'eau est à son maximum (tabl. 3), conséquence, nous l'avons vu, d'une densité algale importante. De plus, la différence de niveau entre été et hiver pour ces populations est plus grande dans la deuxième période de l'expérience, c'est-à-dire lorsque l'implantation algale s'est totalement effectuée.

A l'inverse, nous avons observé en été dans les vases une élévation sensible du nombre de germes/g des populations aérobies ; on rejoint ici les notions plus classiques d'augmentation de la population bactérienne dans les sédiments en fonction de l'élévation de la température décrites par plusieurs auteurs et notamment par COOPER (1953).

Enfin, il est à signaler que dans l'une des expériences effectuées au laboratoire, un éclairage supplémentaire avait été adjoint. Le but de cet aménagement était de favoriser la production d'oxygène dissous dans l'eau et par conséquent de stimuler éventuellement les populations aérobies de microorganismes dans ce milieu. Les comparaisons statistiques entre les résultats obtenus pour ce paramètre n'atteignent pas les seuils de signification; ce dispositif particulier n'a donc pas eu l'efficacité que l'on pouvait espérer (MARTY, 1981)

De même. l'on doit retenir que l'adjonction d'une demi-dose de carbonate de calcium est sans effet perceptible sur les paramètres étudiés.

Après avoir énoncé les principales modifications intervenues au cours de l'expérimentation, nous pouvons à présent essayer d'établir les mécanismes qui régissent ces transformations.

L'apport de carbonate de calcium au niveau des sédiments a pour effet immédiat, d'élever le niveau du $\mathrm{pH}$, de libérer des ions calcium et d'activer la floculation des 
colloides. L'on sait que les bactéries manifestent en général un optimum d'activité entre pH 6 et pH 8 et qu'elles ont besoin, entre autres, pour assurer leur développement, de quantités appréciables de Ca (POCHON, 1958). Il est donc normal qu'à l'augmentation du $\mathrm{pH}$ de la vase et à l'apport d'ions calcium, corresponde une activité microbienne plus intense dans les vases traitées par rapport aux vases témoins.

Cet accroissement des populations de microorganismes va favoriser la dégradation des composés organiques et aboutir ainsi à la minéralisation plus grande de la matière organique ; elle permet la libération de sels minéraux dans l'eau qui participent à la stimulation et à la pousse des herbiers de fond. Ces derniers sous l'effet conjoint de la lumière et de la chaleur augmentent la production d'oxygène dissous dans l'eau, favorisant ainsi les populations aérobies de la phase liquide.

Ainsi se trouve mise en évidence l'influence limitée, mais certaine, du carbonate de calcium sur l'ensemble eau-vase d'un étang.

II semble que l'on puisse retenir l'épandage de craie comme une des solutions possibles au problème de désenvasement d'un étang. On gardera cependant présentes à l'esprit les limites de son action et les caractéristiques propres au milieu traité. En effet, les conclusions énoncées ici concernent particulièrement l'étang de Léon et doivent pouvoir être observées dans des étangs similaires, sans que l'on puisse les extrapoler à des pièces d'eau dont les caractéristiques seraient par trop différentes.

Dans le cas précis de cette étude, et si l'on voulait concilier le coût de l'opération avec l'amélioration sensible obtenue sur la phase liquide (réoxygénation) et sur les sédiments (diminution des composés organiques), l'on pourrait envisager le traitement de cet étang par l'apport d'une quantité de craie équivalente à deux fois la dose de référence.

Cependant, les bilans de fin d'expérimentation font apparaître que les modifications les plus importantes sont constatées dans la couche superficielle de la vase, par conséquent au contact quasi immédiat du carbonate de calcium. II serait donc intéressant de tester l'action de la craie lorsqu'un mélange intime est effectué avec la vase sur une hauteur du sédiment plus importante.

\section{Bibliographie}

ANONYME, 1965. Le Nautex en piscicultures, en rivières, en lacs. OMYA, Doc. 56. MEAC S.A., 28150 Voves, 4 p. polycop.

ASKEL M., 1965. Seasonal quantitative changes in soil fungi. Eesti NSV Tead. Akad. Toim. Biol. seer., 14, 336-376.

BERTRU G., 1975. Etude physico-chimique et microbiologique des échanges sédimentseau : Influence sur la trophie du système et la périodicité du phytoplancton. Thès. Fac. Sci. Univ. Rennes, 243 p.

CAVELIER-JACQUEMAIN C., 1977 a. Influence du déversement de craie dans un milieu limnique eutrophe. J. fr. Hydrol., 8, 2 (23), 75-86.

CAVELIER-JACQUEMAIN C., 1977 b. Traitement du bassin de Sainte Suzanne par la craie en poudre. Thèse, Fac. Sci. Univ. Rennes, 244 p.

CAVELIER C., 1978. Le traitement des eaux eutrophes par la craie. Plaisirs de la Pêche, $179,71-73$.

CHARTRAIN-DEMARQUEZ N., 1977. Sur l'écologie bactérienne et la salubrité des étangs landais. Thèse, Fac. Sci. Univ. Bordeaux I, $199 \mathrm{p}$.

COOPER B.A., 1953. The bottom sediments of lake Lanzo. II. Bacteriological investigations. Revue can. Biol., 12, 457-494.

CTGREF, 1974. Les petits étangs landais ; constat de l'état actuel. Programme de lutte et de prévention contre le comblement, étang de Léon. Etud. Cent. Tech. Génie Rural Eaux For., 10, 16 p. polycopiées.

DEMARQUEZ N., SÉCHET J., 1977. Ecologie bactérienne et salubrité de trois étangs landais. Bordeaux méd., 10, 23, $1609-1619$.

DUCHAUfour Ph., 1965. Précis de Pédologie, 1 vol., 480 p., Masson, Paris

FEUILLET M., GOULEAU D., 1977. Action des épandages de craie (ou $\mathrm{Ca} \mathrm{CO}$ ) sur les vases des claires et des parcs ostréicoles. Rev. Trav. Inst. Pêches marit., 41, 4, 417-436. 
FEUILLET M., GOULEAU D., 1979. Action de l'épandage de carbonate de calcium en poudre sur les vases des parcs à huître de la région de Fouras. Science pêches, 288, 1 6.

GAGNIARD A., 1965. Bilan d'une année "de craie". Truite, Ombre, Saumon, 9, 15-17.

GAGNIARD A., 1966. Bilan d'une année "de craie". Piscic. fr., 6, 31-34.

GAGNIARD A., 1968. La faune et la flore d'une rivière de Haute-Saône reconstituées en six mois après une pollution au cyanure. Plaisirs pêche, 119, 56-58.

GAGNIARD A., 1970. Pourquoi, quand, comment utiliser le Nautex. Plaisirs pêche, 130. 53-55.

GRYGIEREK E., WASILEWSKA B.E., 1979. Possibilité de régulation de biocénoses des étangs à l'élevage des poissons. Bull. Cent. Etud. Rech. sci., Biarritz, 12, 3, 441 442.

HUSSENOT J., 1972. Contribution à l'écologie des eaux stagnantes: Etude de l'étang de Barbillon (Sologne). Mesure de la production bactérienne des eaux. Thès. Fac. Sci. Univ. Orléans. $100 \mathrm{p}$

LAURENT M., SECHET J., 1973. Etude microbiologique de l'influence de la craie sur la vase des étangs. Ann/s Hydrobiol, 4, 2, 143-168.

LEYNAUD G., ARRIGNON J., BARNARD A., LAURENT M., ROFES G., VERNEAUX G., 1969. Possibilités d'amélioration des lacs ou des rivières par immersion de craie. Coll. Biol. Aménagement pêches, Biarritz, 17 p. polycop.

MARTY C., 1972. Etude microbiologique de l'influence du carbonate de calcium sur la vase de l'étang de Léon. Dipl. Etud. Sup. Sci. Nat., Univ. Bordeaux 1, 67 p. polycop.

MARTY C., 1981. La lutte contre l'eutrophisation des étangs landais : action du carbonate de calcium sur les vases de l'étang de Léon. Thèse Fac. Sci. Univ. Bordeaux l, $183 \mathrm{p}$.

NISBET M., VERNEAUX J., 1970. Composantes chimiques des eaux courantes : discussion et proposition de classes en tant que bases d'interprétation des analyses chimiques. Ann/s Limnol., 6, 2, 161-190.

POCHON J., DE BARJAC H., 1958. Traité de microbiologie des sols. Applications agronomiques, 1 vol., 686 p. Dunod. Paris.

POCHON J., TARDIEUX P., 1962. Techniques d'analyse en microbiologie du sol. 1 vol. 112 p., Éditions de la Tourelle, Saint-Mandé.

RAKHNO P.G., 1963. Vest. Akad. Nauk Kazakh. SSR, 10, 54-57.

ROFES G., 1971. Amélioration des étangs ou des rivières par immersion de la craie. Coll. Biol. Aménagement pêches, Biarritz, $15 \mathrm{p}$. polycop.

ROFES G., 1973. Amélioration des étangs ou des rivières par immersion de craie. Coll. Biol. Aménagement pêches, Biarritz, 23 p. polycop.

ROFES G., 1975. Influence de la craie en poudre sur les fonds envasés en eau courante. Bull. Fr. Piscic. 258, 1-14.

SALES L., 1970 a. La craie? Un miracle. Plaisirs Pêche, 129, 19-20.

SALES L., 1970 b. La craie? Un miracle qui persiste. Plaisirs Pêche, 135,12.

SAWYER F., 1964. La craie joue-t-elle un rôle essentiel dans la croissance de la truite. Plaisirs Pêche, 98, 332-338.

SAWYER F., 1965. La craie joue-t-elle un rôle essentiel dans la croissance de la Truite. Plaisirs Pêche, 100, 28-35.

SAWYER F., 1967 a. Nouvelles précisions sur la craie. Plaisirs Pêche, 112, 39.44

SAWYER F., 1967 b. Nouvelles précisions sur la craie. Plaisirs Pêche, 113, 90-92.

SAWYER F., 1967 c. Nouvelles précisions sur la craie. Plaisirs Pêche, 114, 157-159.

SAWYER F., 1967 d. Nouvelles précisions sur la craie. Plaisirs Pêche, 115, 198-202.

STUDER R., 1974. La qualité chimique des eaux des étangs de la Brenne. I.N.R.A. Station d'Agronomie, Châteauroux, $17 \mathrm{p}$. polycop.

VAN BENEDEN G., VAN BENEDEN P., 1966. Le desembouage des lits des rivières par lait de craie et la lutte contre l'eutrophisation. Bull. Cent. Belge Etud. Docum. Eaux, 270. 227-234. 